\title{
Normativas interoperables sobre control de autoridades para bibliotecas digitales semánticas
}

\author{
Fernanda Garzón-Farinós* \\ Fernanda Peset ${ }^{\star \star}$
}

Artículo recibido:

24 de mayo de 2016

Artículo aceptado:

9 de marzo de 2017

\section{RESUMEN}

Las comunidades de la web y las instituciones de conservación del patrimonio han trabajado paralelamente intentando alcanzar un mismo fin: transmitir conocimiento. Las herramientas desarrolladas para bibliotecas intentan ahora confluir con la tecnología de la web semántica. Ambas necesitan expresar instancias con distintos de nombres. Los numerosos esquemas para diferentes usos de las autoridades añaden complejidad. Este trabajo aclara la situación con un cuadro de equivalencias entre nueve normas y facilita la decisión para escoger y convertirlos de uno u otro formato. Ha sido probado en el diccionario biográfico Vestigium.

* Universidad Católica de Valencia San Vicente Mártir, España.

fernanda.garzon@ucv.es

* Universitat Politècnica de València, España. mpesetm@upv.es

INVESTIGACIÓN BIBLIOTECOLÓGICA, vol. 32, núm. 76, julio/septiembre, 2018, México, ISSN: 2448-8321 pp. 167-188 
Palabras clave: Control de Autoridades; Diccionarios Biográficos; Esquemas de Metadatos.

\title{
Interoperable standards on authority control. Trends for semantic digital libraries \\ Fernanda Garzón-Farinós and Fernanda Peset
}

\begin{abstract}
Communities of practice proceeded from web and from cultural heritage institutions worked on parallel lines to achieve the same objective: to transfer the knowledge. The tools developed for libraries are now trying to merge with semantic web technology. Both need to express instances with different forms of names. Numerous schemes for different uses of the authorities add complexity to our study. This paper clarifies the situation by drawing up a table with the equivalences between nine standards. It facilitates the decision to choose one and the exportation to other schemes as well. Vestigium web project, a biographic dictionary, tested our results.
\end{abstract}

Keywords: Name Authority Control; Personal Names; Biographical Dictionaries; Metadata; Semantic Web.

\section{LA ESTANDARIZACIÓN EN EL CONTROL DE AUTORIDADES}

— 1 control de autoridades persigue la identificación unívoca de personas, Wentidades o materias para evitar ambigüedades derivadas de los fenómenos lingüísticos —-sinonimia, homonimia...- o derivadas de las dispersiones debidas a los nombres diferentes por los que se les conoce (Burke y Shorten, 2010: 4-7; Díaz, 2012: 38-40; Dragon, 2009: 186-187; El-Sherbini, 2013: 5; Holm, 1999: 11; Smiraglia, 2004: 292-5; Velluci, 2000: 34-6; Wolverton, 2006: 31-38). Tradicionalmente, ha proporcionado consistencia al catálogo bibliográfico en contenido y forma (Auld, 1982: 322-327; Burger, 1985: 12-27; Clack, 1988: 35-9; Danskin, 1998: 200-201; Dickson y Zadner, 1989: 57-60; Durance, 1993: 38-42; McDonald, 1985: 219-221; Taylor et al., 1985: 195-203; Tillet, 1989: 1-3; Younger, 1994: 133-141). 
Como es lógico, los cambios tecnológicos influyen en el tratamiento de la información, así como en su presentación, almacenamiento y recuperación. El universo es especialmente cambiante, debido al aumento de la diversidad y complejidad de las necesidades de los usuarios y la información (DíazValenzuela et al., 2013: 3994-3995; Leroy y Leffard, 2004: 7-16; Tosaka y Park, 2013: 651).

El mencionado objetivo del control de autoridades ha evolucionado en dos direcciones: 1) mejorar la búsqueda y recuperación de la información por parte de los usuarios y 2) facilitar el intercambio de datos de calidad a escala internacional. En el primer caso, aporta precisión en la recuperación de la información, ya que evita la ambigüedad de los nombres (Díaz-Valenzuela et al., 2013: 3995; Tillet, 2004: 24).

La normalización de los puntos de acceso de personas, organismos o materias aumenta el índice de eficiencia en la recuperación de la información (Díaz, 2012: 36-39; Tillet, 2004: 28). A medida que aumenta el volumen de la información, el control de autoridades es cada vez más necesario (Díaz, 2012: 36; Leiva-Mederos et al., 2013: 536-537). En el segundo caso, el control de autoridades se enfrenta a los retos derivados del intercambio de datos en entornos automatizados, en los que el usuario desea acceder a los registros en su lengua, independientemente del idioma o escritura de origen (Ferran, 2009; Harper y Tillet, 2007: 56-59; Kimura, 2014: 733-737; Tillet, 2004: 32-39). En este sentido, se han desarrollado múltiples protocolos de comunicación que trabajan la interoperabilidad entre las fuentes de datos (Gorman, 2004: 1520; Leiva-Mederos et al., 2013: 536-542). Entre ellos destacan los que se aplican a la web semántica.

En el camino para llegar a la web semántica y a los protocolos automatizados fue necesario construir antes estándares de descripción —sean para objetos, personas o materias-, pues son los que proporcionan consistencia a la metainformación. Dado que las bibliotecas y archivos llevan siglos de tradición organizando el conocimiento, el World Wide Web Consortium (W3C) finalmente valoró su experiencia. Ciertas normas bibliotecarias y archivísticas efectúan el control de autoridades, por ejemplo, la ISAAR (CPF) para los Registros de Autoridad de Archivos relativos a instituciones, personas y familias. Otras han servido de base para desarrollar lenguajes y estándares que permiten codificar metadatos e intercambiar registros de autores —Encoded Archival Context-Corporate Bodies, Persons and Families (EAC-CPF) - . Y otras se utilizan para relacionar entre sí los diferentes estándares en la web, como el Resource Description Framework (RDF).

En este momento, la comunidad bibliotecaria y las últimas tendencias en recuperación de la información a través de la web están confluyendo. Para 
ello es necesario que los estándares se armonicen, especialmente en el área del control de autoridades.

El objetivo de este estudio es conocer a profundidad la normativa para los autores personales. Realiza una revisión de sus tradiciones, punto de partida necesario para enriquecer el escenario actual, en el que aparece este tipo de información, bibliotecas y diccionarios biográficos. Nuestro trabajo aporta un cuadro con las equivalencias entre las nueve normativas más significativas. Supone el estudio previo que necesita cualquier desarrollo tecnológico para que su apartado de información biográfica sea compatible con los presupuestos de la web semántica. Este trabajo ha sido testado para confeccionar las biografías de personajes en la biblioteca digital Vestigium, orientada a recopilar la información de humanistas y científicos del siglo XX. Vestigium es ahora un proyecto financiado por el gobierno regional valenciano (Prometeo). Puede verse su apariencia actual en <http://www.vestigium.es/biblioteca/index.php >.

\section{Material y MÉTODO}

Se revisaron todos los sistemas de codificación a escala nacional e internacional para bibliotecas y archivos, y algunos estándares propuestos para la web semántica en el control de autoridades de nombres de persona, entidad, título uniforme, materia, género/forma, tanto a nivel de concepto, visualización, estructura, intercambio de información, como sobre metadatos. La búsqueda se realizó en los principales organismos de normalización. En el área de archivos se consultó el Consejo Internacional de Archivos (CIA, o ICA por sus siglas en inglés, 2004).

En el área de bibliotecas se visitó la sección "Standards" de la Library of Congress (LC, 2015), como fuente de la normativa para registros bibliográficos; el website de la International Federation of Library Associations and Institutions (IFLA, 2016), así como las secciones de "Normas y estándares de catalogación” (Biblioteca Nacional de España, 2016a) y "Organismos de normalización” de la Biblioteca Nacional de España (2016b). Respecto de la localización de recomendaciones de la web semántica, se consultó el apartado “Semantic Web” del W3C (2015), seleccionando las que están estrechamente relacionadas con datos de autoridad de persona.

De todas las normas recuperadas, se analizaron únicamente las referentes al control de autoridades de personas y sus relaciones con instituciones, 
personas y recursos de información externos. Esta revisión de normativa se completó con bibliografía, especialmente para localizar los casos de aplicación en la Web of Science de Thomson Reuters.

La información extraída de todas las normas y estándares seleccionados se trató de forma global, sintetizando y siguiendo la misma estructura para analizar las relaciones y equivalencias entre cada una. Tras este análisis, se fue necesario elaborar un cuadro que contemplara las conversiones y equivalencias.

\section{Algunas normativas}

Veamos detenidamente las más significativas. ISAAR (CPF) se utiliza como guía para la redacción de registros de autoridad de archivos referentes a instituciones, personas y familias, entendidos como productores de la documentación que ha de gestionarse. Si bien nace para la comunidad archivística, prevé la posibilidad de compartir y vincular datos e información con los catálogos y sistemas descriptivos, más allá de ese ámbito (CIA, 2004). Se trata de una norma que destaca las relaciones que afectan a estas autoridades, lo que conforma la esencia de la web semántica.

Las "Directrices para registros de autoridad y referencias" (GARR) proporcionan las directrices para la visualización de los registros de autoridad y referencias para nombres de persona y títulos uniformes, facilitando el intercambio internacional en sistemas informáticos y de forma impresa (Buizza, 2004: 126; CIA, 2004; Knight, 2005).

A principios del siglo XXI, la comunidad bibliotecaria efectuó una transición en sus normas. Los procesos de automatización del siglo XX habían forzado los sistemas informáticos para que aceptaran normas concebidas para entornos manuales. Para corregir la disfunción entre la capacidad de la tecnología para recuperar datos y las normas utilizadas, se redactaron los Functional Requeriments of Bibliographic Records (FRBR), de los que derivará la norma internacional Resource Description and Access (RDA). Los Functional Requirements for Authority Data (FRAD) aparecen sobre la base de los FRBR, GARR, Unimarc Manual-Authorities Format, y Mandatory Data Elements for Internationally Shared Resource Authority Records (MLAR).

Esta transición, conocida como Bibliographic Framework Iniciative (Bibframe), conduce a IFLA a desarrollar un modelo conceptual para los registros de autoridad más cercano a cómo la comunidad de informáticos entiende las necesidades de los usuarios. Sobre esa base proporcionan los requisitos que necesita un sistema para intercambiar autoridades a escala internacional (Patton, 2009, 1). Marc 21 Conciso para Datos de Autoridad 
es otro referente de la familia Marc para la estructura y forma común de las autoridades de nombres personales y corporativos, títulos uniformes, materias y series. Se considera una herramienta imprescindible para el intercambio de registros de autoridades en entornos automatizados (LC, 2016b; Lam, 2002: 88; Veve, 2009: 43-5). Bibframe pretende ser un modelo para los futuros recursos y fondos bibliográficos codificados en Marc 21, adaptado a los requerimientos de la web actual. El resultado aprovecha la web como una arquitectura subyacente que da forma a un marco descriptivo común con datos enlazados (Linked Data o LD), ofreciendo un modelo flexible tanto para datos bibliográficos, como de autoridades (LC, 2016a; Mitchell, 2013: 27-33).

\section{Estudio y armonización de las diferentes normativas}

Tal y como hemos visto, existen multitud de normas que son aplicadas a diferentes necesidades o comunidades de práctica. Las relacionadas con el mundo bibliotecario o archivístico cuentan con años de tradición; mientras que las relacionadas con la web, obviamente, son de reciente creación. Además, a veces son recomendaciones que alcanzan, o no, una amplia implantación, pero no se comparan con normas de rango casi de estándar internacional — caso de la CDU - que son las que acostumbran a usarse en las instituciones documentales. No es comparable el esfuerzo invertido en normas como rda con otros esquemas de descripción más simplificada que se aplican en el mundo web.

Ésta es la razón por la que este apartado se estructura tomando como base las normas más clásicas, que se hacen equivaler con las del ámbito web, las cuales en ocasiones no recogen tantas propiedades.

A partir del modelo conceptual frad se trazaron los atributos y las relaciones de personas, de nombres de persona y de puntos de acceso controlado.

\section{Conversiones y equivalencias entre normativas}

Los cuadros 1 a 3 presentan la aportación de este trabajo en el ámbito teórico, conceptual, de la Biblioteconomía. Reflejan y aclaran las equivalencias y conversiones de nueve normativas, lo que hasta ahora era inédito: FRAD, ISAAR (CPF), EAC-CPF, GARR, Marc 21, MADS, Dublin Core RDF, Marc XML y FOAF. Como dijimos, los estándares del sector de la gestión de información tradicional —archivos y bibliotecas — son más profusos que los que se utilizan en el entorno web, como Dublin Core o FOAF. 
En el cuadro 1 se muestran los atributos de la Entidad persona de FRAD y sus conversiones a otros estándares de archivos, bibliotecas y web semántica. Cabe destacar que los atributos de persona de frad fechas asociadas a la persona, título de la persona, género, lugar de nacimiento y fallecimiento... coinciden con los nuevos campos Marc 21 que atienden las necesidades de RDA. Como es bien sabido en la comunidad bibliotecaria, la manera más eficiente de establecer diferencias entre homónimos es aportar datos sobre las personas que se refiera a esos aspectos esenciales.

Por ejemplo, mediante los códigos de subcampo de Lugar asociado de Marc 21 (370) es posible incluir todos los lugares de la persona biografiada, tal y como refleja el cuadro 1 . Entre los cuales encontramos el Lugar de nacimiento ( 370 \$a), el Lugar de fallecimiento (370 \$b), el País (370 \$c) o el Lugar de residencia $(370 \$ \mathrm{e})$.

Por otro lado, el cuadro 2 reúne las equivalencias de los Atributos de nombre y de Puntos de acceso controlado. Su función es el control de la forma del nombre, que constituye la segunda gran tarea del trabajo con autoridades. Se organiza tomando también como base FRAD, puesto que simplifica el complejo mundo de las autoridades en dos únicos atributos: Tipo de nombre y Fuente de acceso controlado, lo que permite incluir las fuentes consultadas para elaborar los registros de los autores.

Por último, las relaciones entre las autoridades también se representan en todas estas normativas. Su objetivo es intentar mantener unidas las variantes que se producen a lo largo del tiempo, como nombres anteriores o posteriores (especialmente en corporativos), o variantes de nombres al utilizar otros idiomas, otras escrituras alternativas u otras reglas.

Las relaciones más comunes son de lengua paralela, de escritura alternativas y de diferentes reglas. La primera columna del ejemplo detalla las relaciones a nivel general entre los tipos de entidad, siguiendo la estructura de FRAD: Relación entre persona y persona, la Relación entre persona y familia y Relación entre persona y entidad corporativa. Para aclararlo con brevedad, se ofrece el ejemplo de Alfonso Reyes Ochoa desde FRAD a Marc 21, como ayuda a quienes tengan que desarrollar una aplicación que refleje las relaciones de personas en cuanto a nombres de personas, familias y entidades: 


\begin{tabular}{|c|c|c|c|}
\hline 놀 & 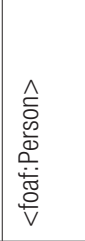 & 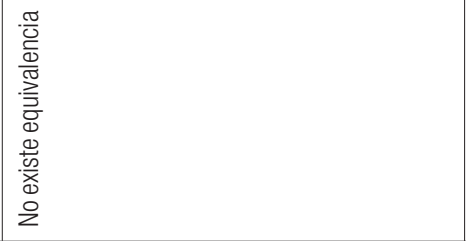 & 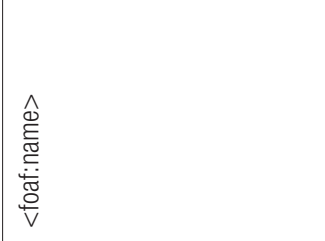 \\
\hline 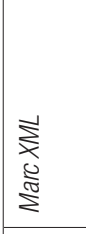 & 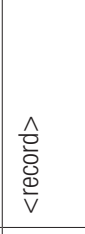 & 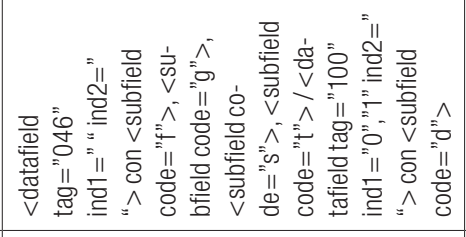 & 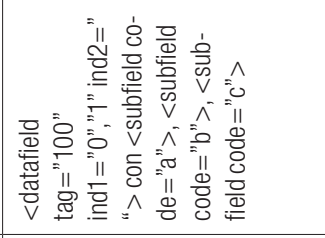 \\
\hline 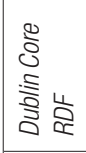 & 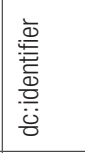 & 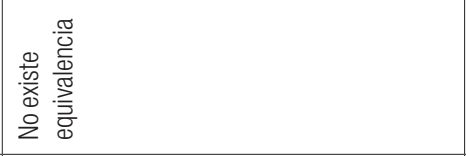 & 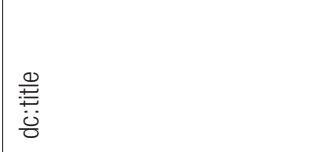 \\
\hline 点 & 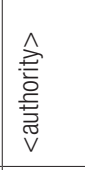 & 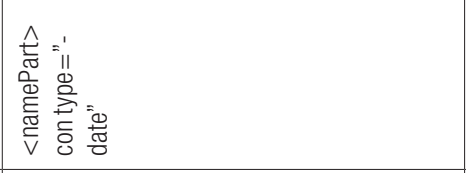 & 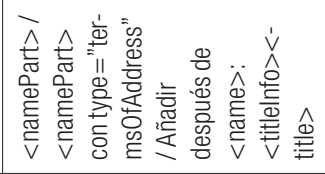 \\
\hline \begin{tabular}{l}
$\bar{N}$ \\
$\mathbb{J}$ \\
\multirow{2}{*}{} \\
\end{tabular} & ஜ̊ & $\begin{array}{l}0 \\
0 \\
0 \\
0 \\
0 \\
0 \\
0 \\
\end{array}$ & 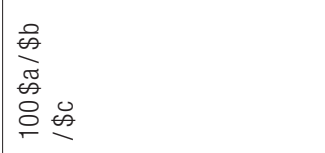 \\
\hline $\begin{array}{l}\frac{x}{2} \\
\frac{2}{5} \\
心\end{array}$ & 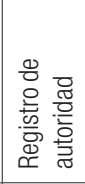 & 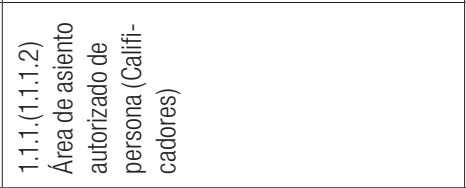 & 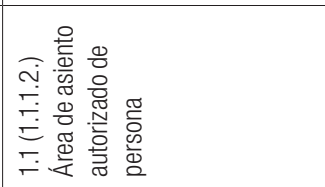 \\
\hline 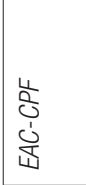 & 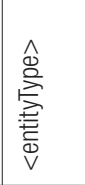 & 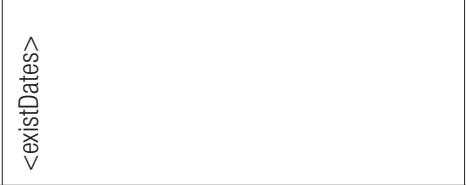 & 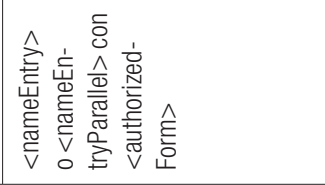 \\
\hline $\begin{array}{l}\frac{\pi}{2} \\
\frac{1}{0} \\
\frac{2}{3} \\
\frac{3}{5} \\
\end{array}$ & 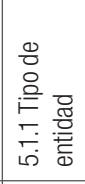 & 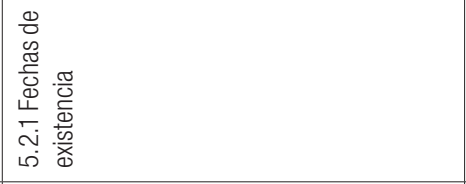 & 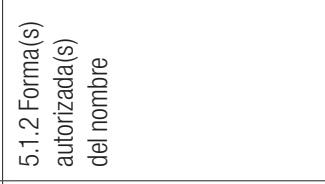 \\
\hline 㴖 & 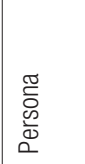 & 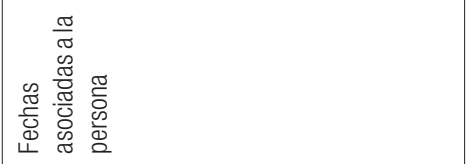 & 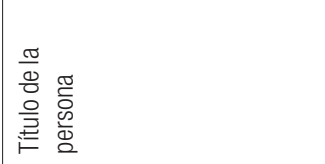 \\
\hline
\end{tabular}




\begin{tabular}{|c|c|c|c|c|c|}
\hline 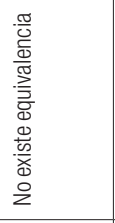 & 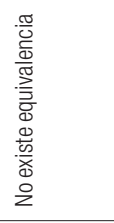 & 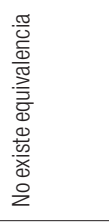 & 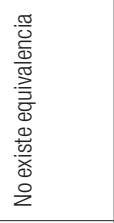 & 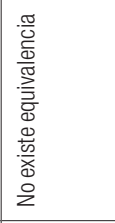 & 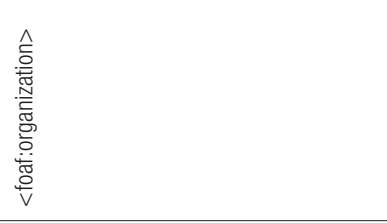 \\
\hline 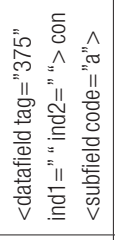 & 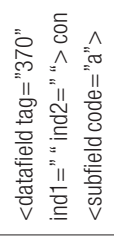 & 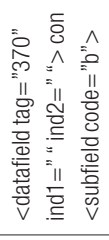 & 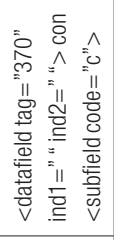 & 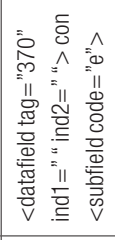 & 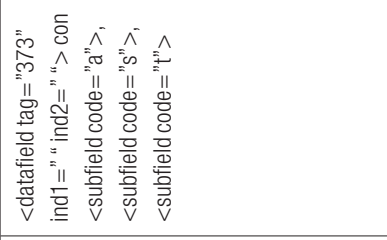 \\
\hline 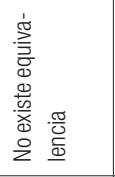 & 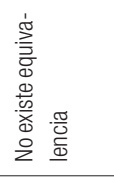 & 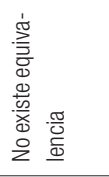 & 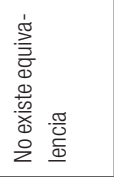 & 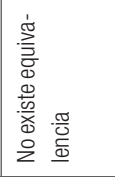 & 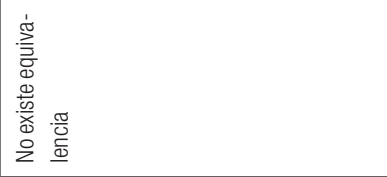 \\
\hline 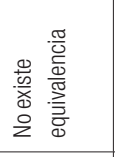 & 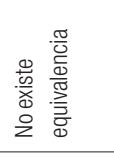 & 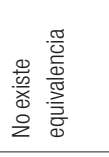 & 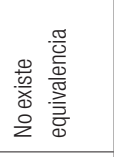 & 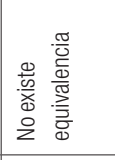 & 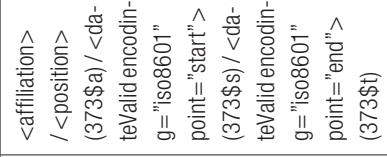 \\
\hline $\begin{array}{l}\mathbb{N} \\
\stackrel{\infty}{\infty} \\
\stackrel{\infty}{\infty}\end{array}$ & 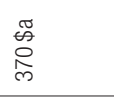 & $\begin{array}{l}\text { के } \\
\text { 尺े } \\
\text { ले }\end{array}$ & $\begin{array}{l}0 \\
\stackrel{0}{\circ} \\
\text { 尺े }\end{array}$ & 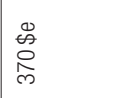 & $\underset{\infty}{\infty}$ \\
\hline 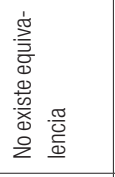 & 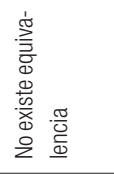 & 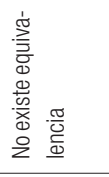 & 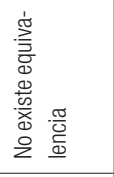 & 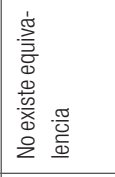 & 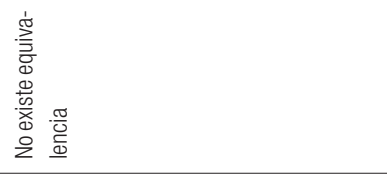 \\
\hline $\begin{array}{l}\hat{\hat{E}} \\
\text { 言 } \\
\text { 产 } \\
\text { 迎 } \\
\text { v }\end{array}$ & 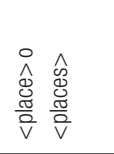 & 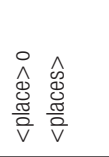 & 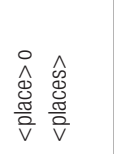 & 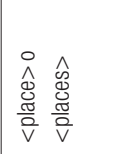 & 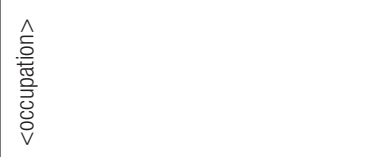 \\
\hline 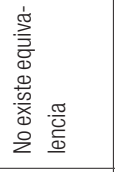 & 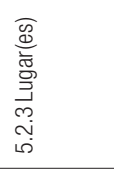 & 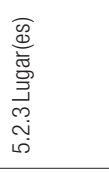 & 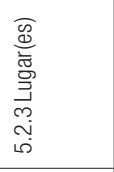 & 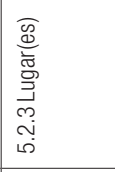 & 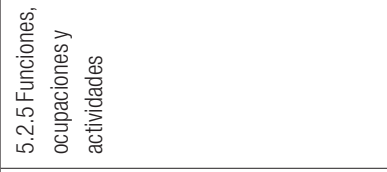 \\
\hline 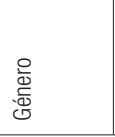 & 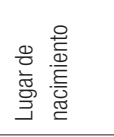 & 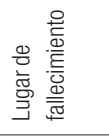 & $\frac{\infty}{\bar{\sigma}}$ & 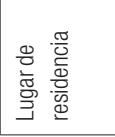 & 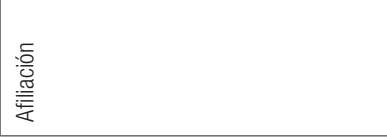 \\
\hline
\end{tabular}




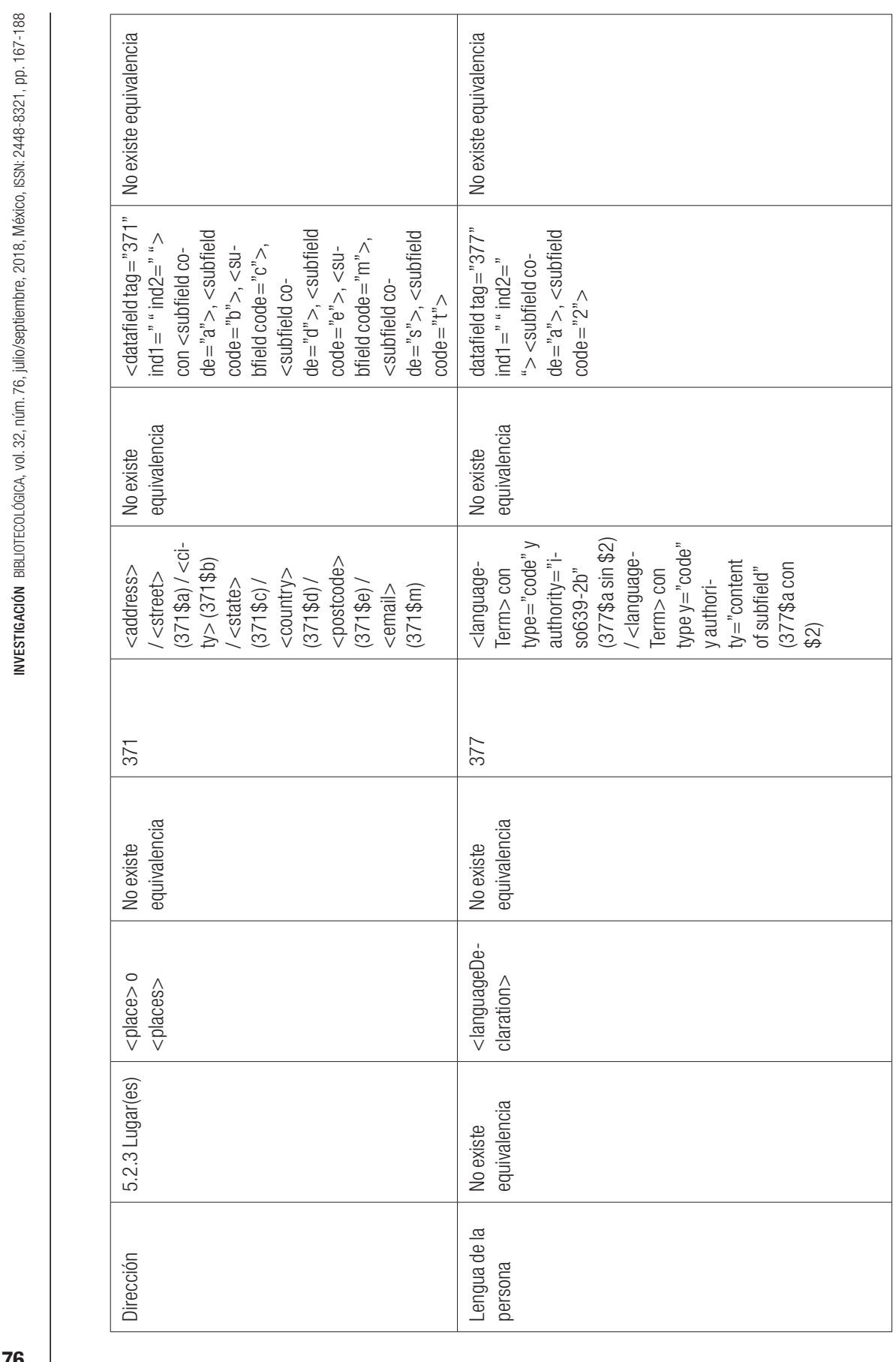


NORMATIVAS INTEROPERABLES SOBRE CONTROL DE AUTORIDADES...

\begin{tabular}{|c|c|}
\hline 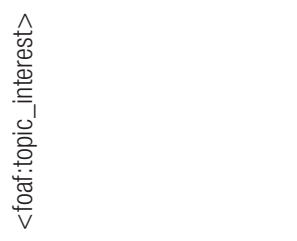 & 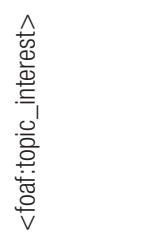 \\
\hline 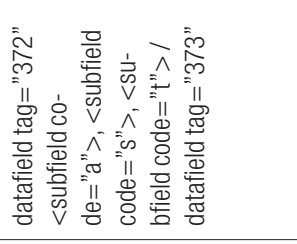 & 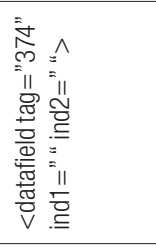 \\
\hline 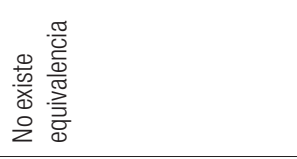 & 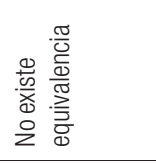 \\
\hline 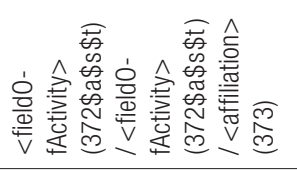 & 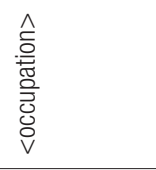 \\
\hline 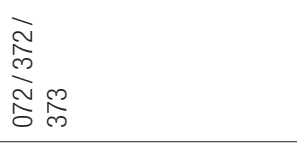 & $\underset{\text { d }}{\mathbb{N}}$ \\
\hline 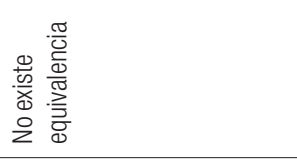 & 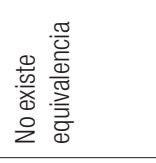 \\
\hline 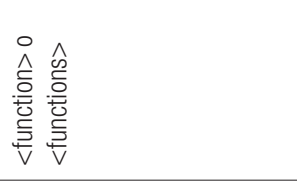 & 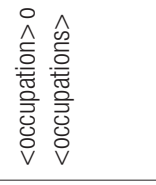 \\
\hline 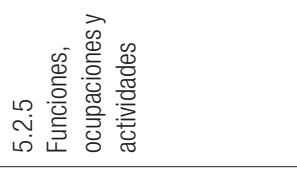 & 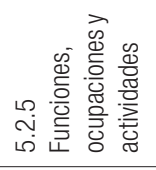 \\
\hline 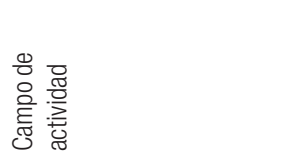 & 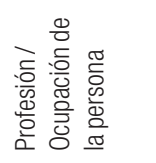 \\
\hline
\end{tabular}

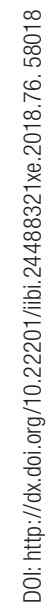




\begin{tabular}{|c|c|}
\hline 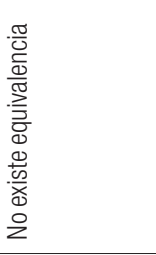 & 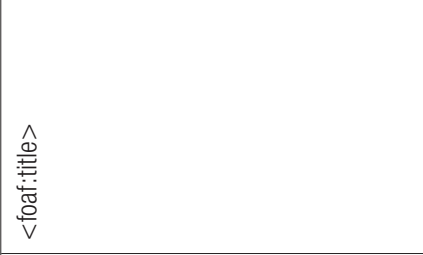 \\
\hline 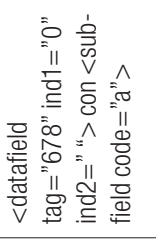 & 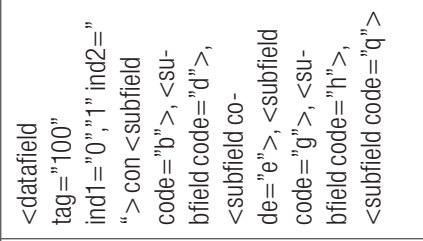 \\
\hline 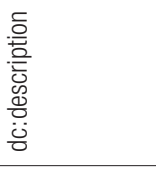 & 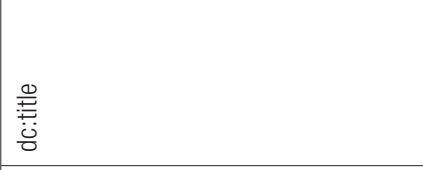 \\
\hline 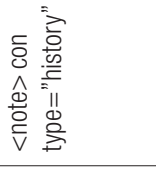 & 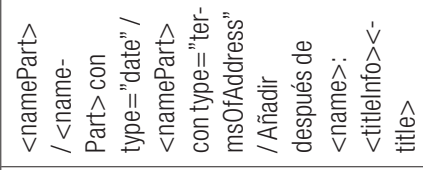 \\
\hline$\stackrel{\infty}{0}$ & 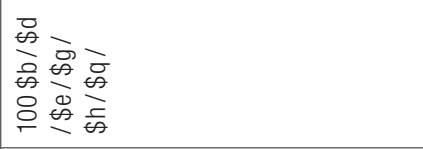 \\
\hline 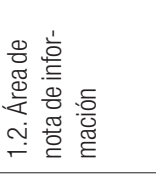 & 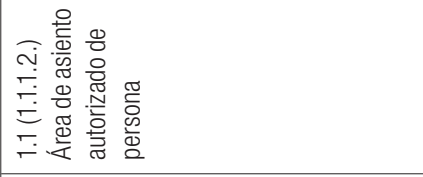 \\
\hline 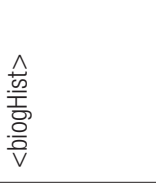 & 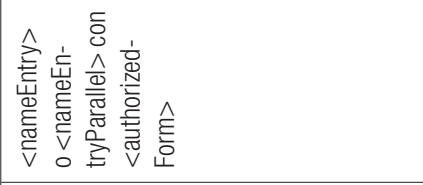 \\
\hline 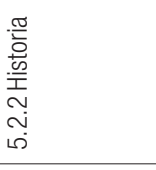 & 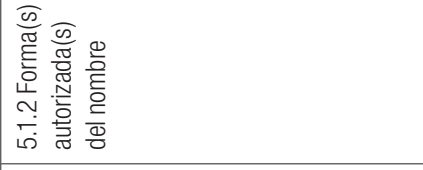 \\
\hline 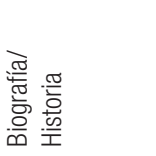 & 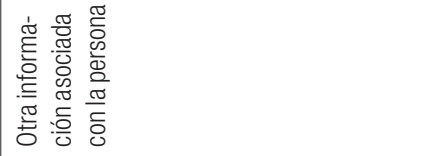 \\
\hline
\end{tabular}




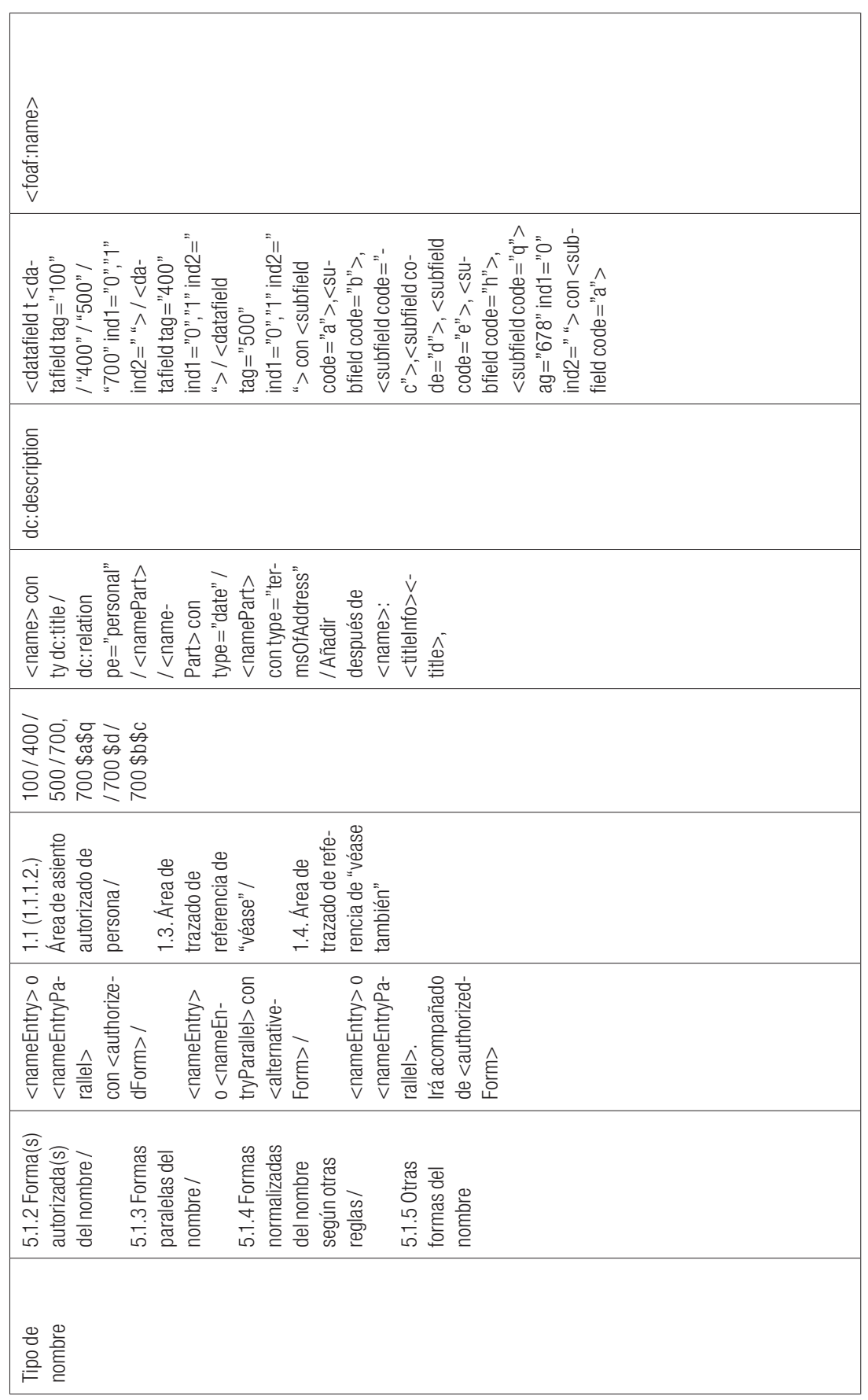




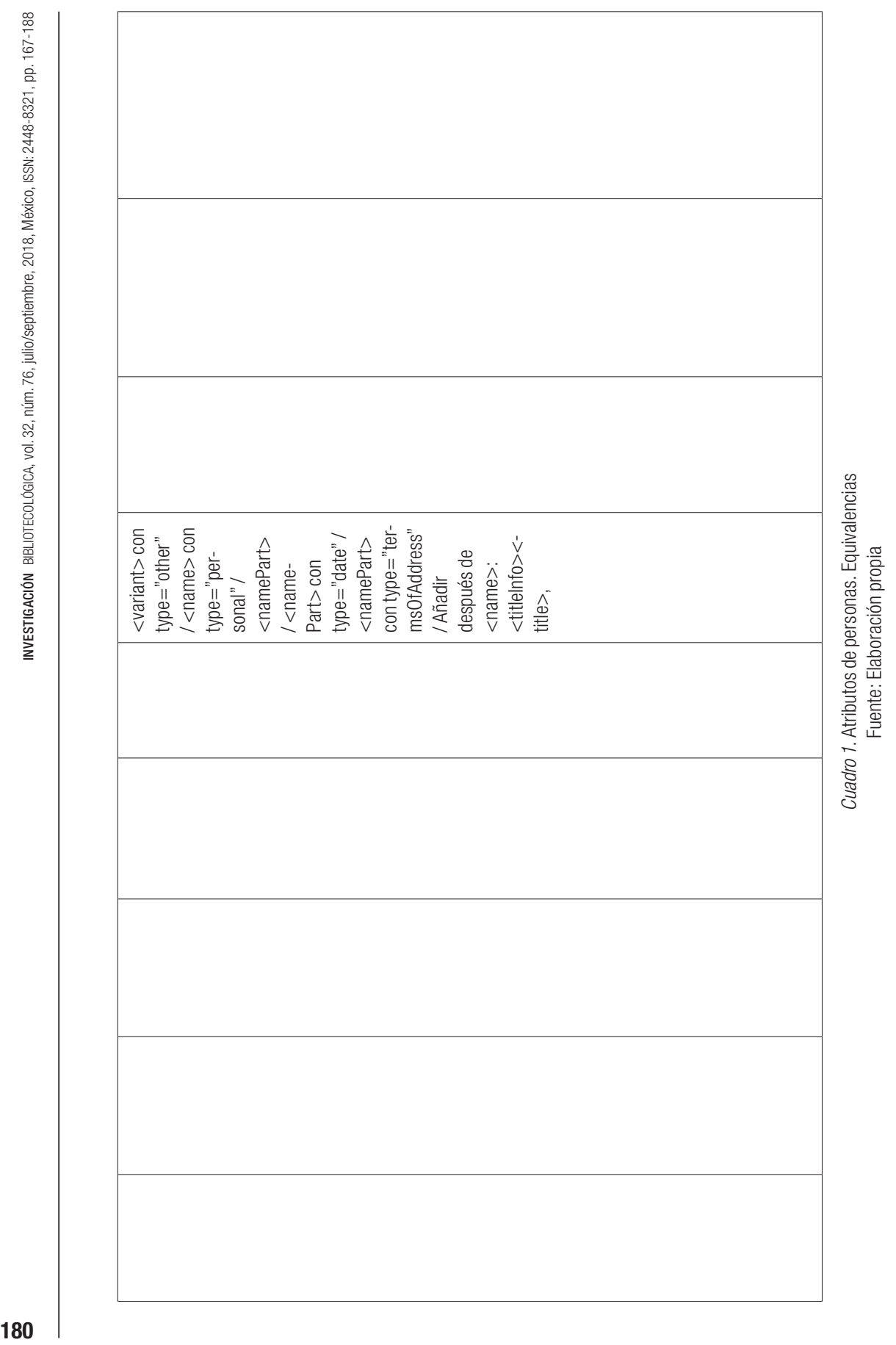




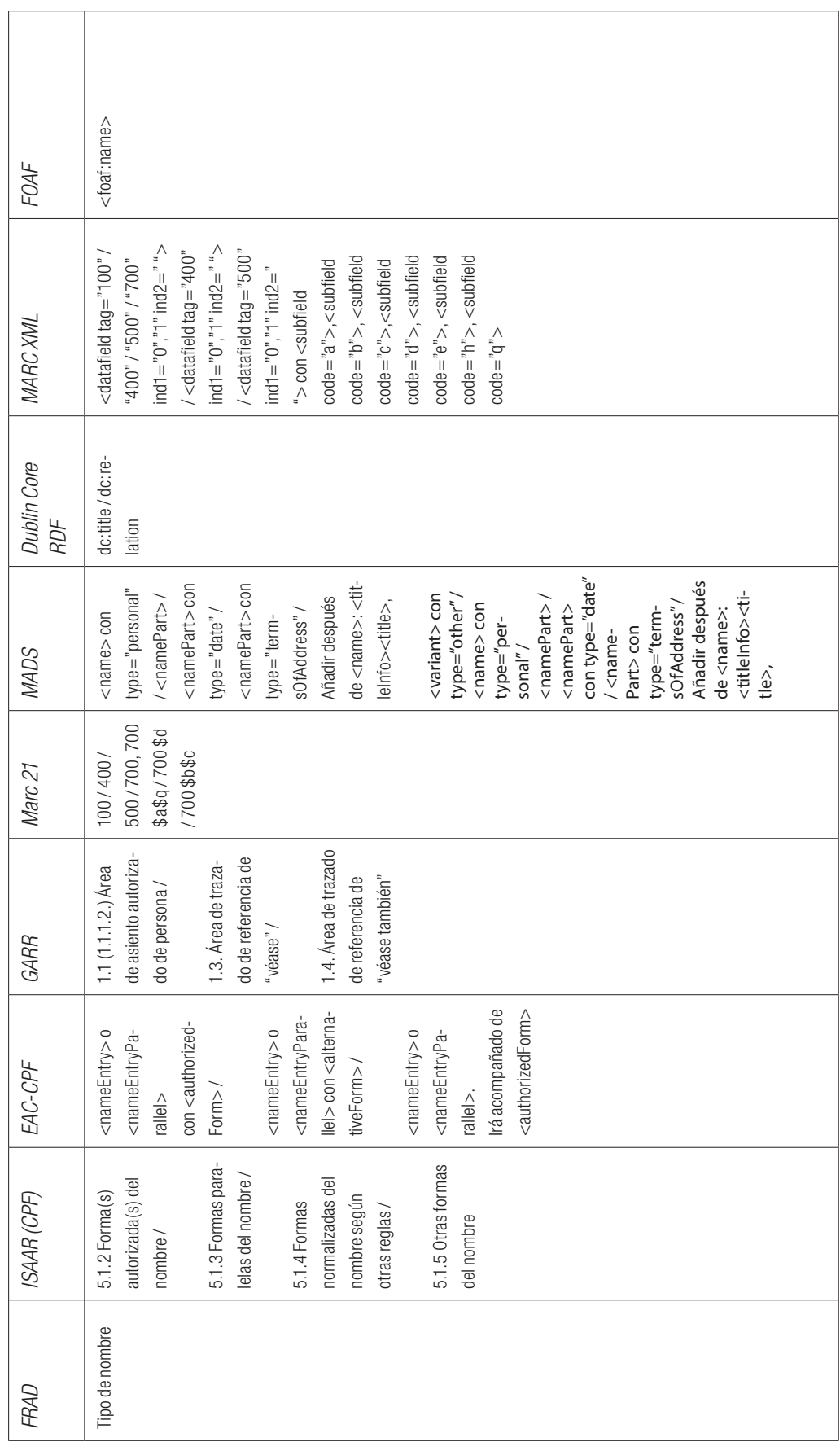




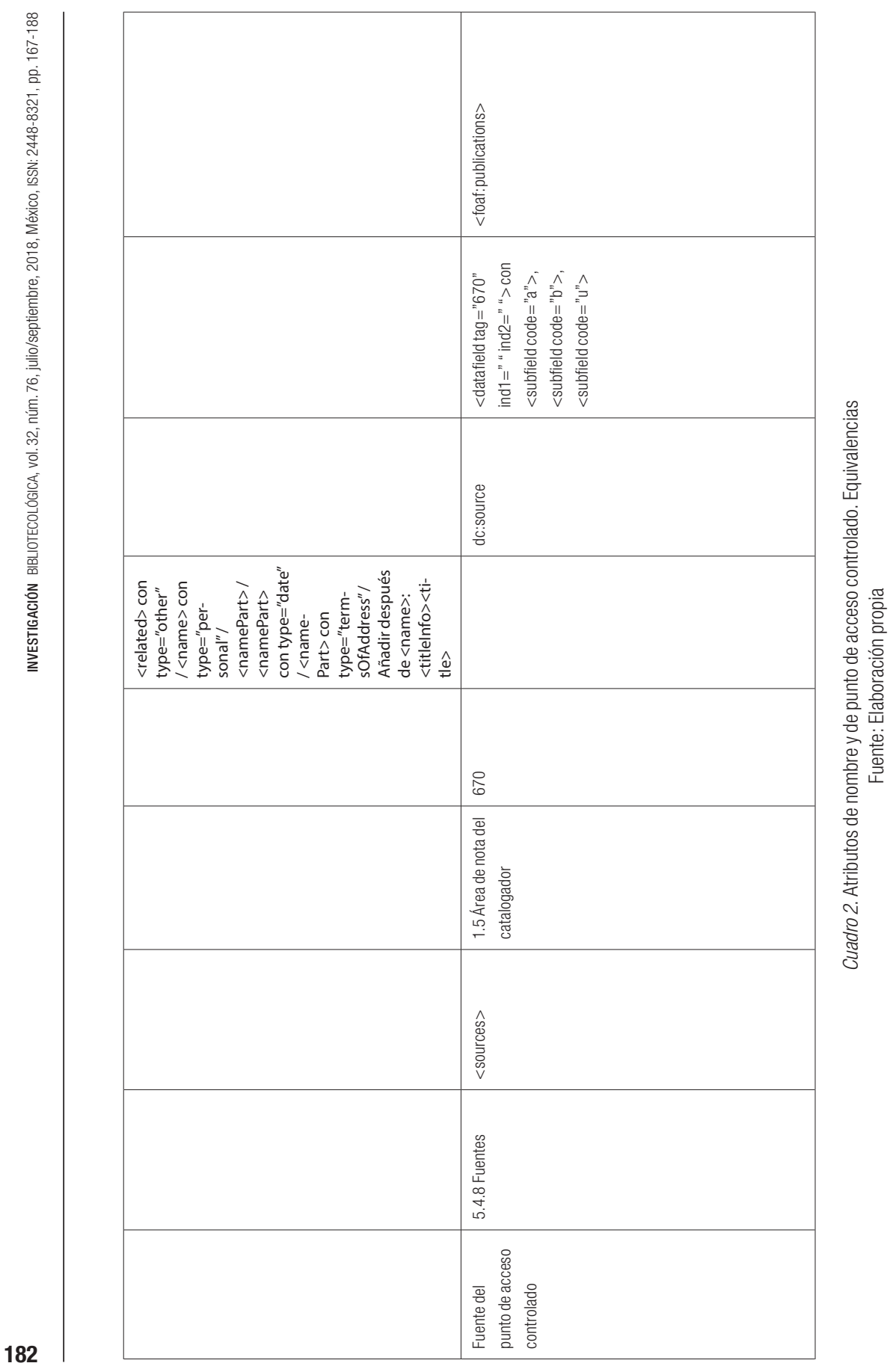




\begin{tabular}{|c|c|}
\hline $\begin{array}{l}\text { Tipo de relación } \\
\quad F R A D\end{array}$ & Código Marc 21 \\
\hline Persona a persona y familia & $\begin{array}{l}5001 \$ \text { aReyes Ogazón, Bernardo } \\
\text { Dorote0\$4http://purl.org/vocab/relationship/child0f\$iHijo de } \$ w i \\
5001 \$ a 0 \text { choa de Reyes, } \\
\text { Aurelia } \$ 4 \text { http://purl.org/vocab/relationship/childOf\$iHijo de } \$ w i \\
5001 \$ \text { aMota, Manuela } \$ 4 \text { http://purl.org/vocab/relationship/spouseOf } \\
\$ i \text { Esposo de } \$ w i\end{array}$ \\
\hline \multirow[t]{2}{*}{ Persona a institución } & $\begin{array}{l}5101 \$ a \text { Ateneo de la Juventud Mexicana } \$ d 1909 \text { \$iFundador de } \\
\$ 4 \mathrm{http}: / / \text { dbpedia.org/ontology/leaderTitle } \$ \text { wi }\end{array}$ \\
\hline & $\begin{array}{l}5101 \text { \$aEscuela Nacional de Altos Estudios, actual Facultad de Filosofía } \\
\text { y Letras (Universidad Nacional Autónoma de México) } \$ d 1912 \$ i \\
\text { Secretario de } \$ 4 \mathrm{http}: / / \text { dbpedia.org/ontology/leaderTitle } \$ w i\end{array}$ \\
\hline
\end{tabular}

Cuadro 3. Relaciones de personas en cuanto a nombres, familias y entidades Fuente: Elaboración propia

\section{Conclusiones}

Las comunidades profesionales asociadas a la web semántica y a las instituciones de la memoria bibliotecas, archivos y museos (LAM) han trabajado durante años en paralelo, si bien intentaban alcanzar un mismo fin, la transmisión de conocimiento. Entre los objetivos concretos en los que coinciden, apreciamos la necesidad de nombrar conceptos, nombrar entidades y, con esto, ofrecer las diferentes formas en que pueden aparecer los nombres. Las LAM se han ocupado, tradicionalmente, del control de la información, y entre ello de las autoridades especialmente. Sin embargo, la aparición de la web diluye las formas tradicionales del control de autoridades (Garzón, 2015: 196-265). Por este motivo, existe la necesidad de estudiarlas, ya que las autoridades son entidades abstractas que aparecen en múltiples entornos de uso. Su estudio y limitación resulta complejo, ya que en cada contexto las autoridades son tratadas de forma diferente.

La web semántica es un intento de mejorar la recuperación de la información e interoperabilidad entre máquinas, utilizando el marco de descripción RDF. No obstante, este marco sigue necesitando normas concretas, por lo que las procedentes de la comunidad de las LAM son reconocidas como especialmente valiosas: normas de descripción, vocabularios controlados, clasificaciones, etc. Los registros de autoridad cumplimentados con FRAD y RDA ofrecerán información contextualizada. 
A partir de las relaciones establecidas, y siguiendo el modelo de los LD, los usuarios navegarán por los elementos digitales que aparecen en los registros de autoridad, conectando con otros de tipología idéntica o distinta, provenientes de fuentes externas, lo que permite enriquecer los datos iniciales.

Este trabajo de investigación proporciona las equivalencias entre normativas sobre autoridades de persona, demostrando que es posible que el mundo bibliotecario y el mundo web confluyan con relativa facilidad. De esta manera, los sistemas de información sobre autores, tanto bibliotecas como diccionarios biográficos, estarán en consonancia con la Agenda Digital para Europa (European Comission, 2010) y el desarrollo de la Estrategia Europea 2020 (European Comission, 2016), que promueve la aplicación de estándares interoperables en la Unión Europea.

\section{Agradecimientos}

Este trabajo, como parte de Vestigium, ha sido financiado por la UCV 2011011-011, Generalitat Valenciana; Conselleria de Educación. Redes de excelencia Prometeo 2013/041; MICINN. Subprograma de Acciones Complementarias para los Proyectos de Investigación Fundamental no Orientada. CSO2011-13730-E. Está basado en la tesis de una de las autoras

\section{REFERENCIAS}

Auld, Larry. 1982. "Authority control: An eighty-year review". Library Resources and Technical Services 26:319-30.

Biblioteca Nacional de España. 2016a. "Normas y estándares de catalogación". $<$ http://www.bne.es/es/Inicio/Perfiles/Bibliotecarios/Procesos-tecnicos/ NormasInternacionales/>, actualizada en enero de 2016, consultada el 11 de marzo de 2016.

Biblioteca Nacional de España. 2016b. "Organismos de normalización". <http://www. bne.es/es/Inicio/Perfiles/Bibliotecarios/OrganismosNormalizacion/index.ht$\mathrm{ml}>$, actualizada en enero de 2016, consultada el 15 de febrero de 2016.

Buizza, Pino. 2004. "Bibliographic control and authority control form Paris principles to the present". Cataloging \& Classification Quartely 38: 117-133.

Burger, Robert H. 1985. Authority work: the creation, use, maintenance, and evaluation of authority records and files. Littleton: Libraries Unlimited.

Burke, Susan K. y Jay Shorten. 2010. "Name authority work today: a comparison of types of academic libraries". Library Resources and Technical Services 54: 4-20.

Clack, Doris H. 1988. "Authority Control and Linked Bibliographic Databases". Cataloging and Classification Quartely 8:35-46.

Consejo Internacional de Archivos (CIA). 2004. ISAAR: Norma internacional sobre los registros de autoridad de archivos relativo a instituciones, personas y familias. Versión española de M. Elena Cortés Ruiz y Blanca Desantes Fernández. 
Madrid: Ministerio de Cultura, Secretaría General Técnica. $<$ http://www.archivonacional.go.cr/pdf/ISAAR2-ES.pdf>, consultada el 5 de octubre de 2014.

Danskin, Alan. 1998. "International initiatives in authority control". Library Review 47: 200-205.

Díaz Rodríguez, Yoselyn. 2012. "Control de autoridades de nombres personales de autores cubanos en ciencias de la salud". Acimed 23:35-44.

Díaz-Valenzuela, Irene, María J. Martin-Bautista, María-Amparo Vila y Jesús R. Campaña. 2013. "An automatic system for identifying authorities in digital libraries". Expert Systems with Applications 40: 3994-4002.

Dickson, Jean y Patricia Zadner. 1989. "Authority control and the authority file: a functional evaluation of LCNAF on RLIN". Cataloging and Classification Quarterly 9: 57-73.

Dragon, Patricia M. 2009. "Name Authority Control in local digitalization projects and the Eastern North Carolina Postcard Collection". Library Research and Technological Services 53: 185-196.

Durance, Cynthia J. 1993. "Authority control: beyond a bowl of alphabet soup". Archivaria 35 (primavera): 38-46.

El-Sherbini, Magda. 2013. "Bibliotheca Alexandrina's model for Arabic Name Authority Control”. Library Resources and Technical Services 57: 4-17.

European Comission. 2016. "Europe 2020”. <http://ec.europa.eu/europe2020/europe-2020-in-a-nutshell/index_en.htm>, actualizada el 15 de julio de 2016, consultada el 25 de julio de 2016.

European Comission. 2010. "Digital agenda for Europe”. <http://eur-lex.europa.eu/ legal-content/EN/TXT/?URI=URISERV:si0016>, actualizada el 25 de junio de 2010, consultada el 13 de octubre de 2014.

Ferran, Inma. 2009. "El catàleg d'autoritats de noms i títols de Catalunya (CÀNTIC)". Bid: textos universitaris de biblioteconoia $i$ documentació, no. 22. $<$ http://bid.ub. edu/22/ferran.htm>, consultada el 23 de junio de 2014.

Garzón Farinós, María Fernanda. 2015. El registro de autoridades personales tras la aparición del web. València: Universitat Politècnica de València <http://hdl. handle.net/10251/46972>, consultada el 23 de febrero de 2016.

Gorman, Michael. 2004. "Authority control in the context of bibliographic control in the electronic environment”. Cataloging and Classification Quarterly 38: 11-22.

Harper, Corey A. y Barbara B. Tillet. 2007. "Library of Congress controlled vocabularies and their application to the semantic web". Cataloging and Classification Quarterly 43: 47-68.

Holm, Liv Aasa. 1999. "Authority control in an international context in the new environment”. ICBC 28: 11-13.

IFLA. 2016. "International Federation of Library Associations and Institutions". <http://www.ifla.org/about>, actualizada el 29 de julio de 2016, consultada el $1^{\circ}$ de agosto de 2016.

Kimura, Maiko. 2014. "Differences in representations of Japanese name authority data among cjk countries and the Library of Congress". Information Processing and Management 50: 733-751.

Knight, Bonnie M. 2005. "The evolution of FRAR and the Future of Authority Control”. San José, Calif.: ponencia presentada en la San Jose State University School 
of Library and Information Science L249. <http://www.informedge.net/FRAR. pdf $>$, consultada el 23 de junio de 2014.

Lam, Ki-Tat. 2002. "XML and global name access control". OCLC Systems and Services 18: 88-96.

Leiva-Mederos, Amed, José A. Senso, Sandor Domínguez-Velasco y Pedro Hípola. 2013. "Authoris: a tool for authority control in the semantic web". Library Hi Tech 31: 536-553.

Leroy, Sarah Y. y Thomas S. Leffard 2004. "Impact of web access on cataloging". Cataloging and Classification Quarterly 38: 7-16.

McDonald, David R. 1985. "Data dictionaries, authority control, and online catalogs: a new perspective”. The Journal of Academic Librarianship 11: 219-222.

Mitchell, Erik T. 2013. "Library Linked Data: Research and Adoption. Three case studies in linked Open Data". Library Technology Reports 49: 26-43.

Patton, Glenn E, ed. 2009. Functional Requeriments for Authority Data: A Conceptual Model. Múnich: K.G. Saur. <http://www.ifla.org/files/assets/cataloguing/frad/ frad_2009-es.pdf>, consultada el 23 de marzo de 2014.

Smiraglia, Richard P. 2004. "Authority Control of Works: cataloging's chimera?". Cataloging and Classification Quarterly 29: 291-308.

Taylor, Arlene G., Margaret F. Maxwell y Carolyn O. Frost. 1985. “Network and vendor authority systems". Library Resources and Technical Services 29: 195-205.

The Library of Congress. 2016a. "Bibframe: Bibliographic Framework Iniciative". $<$ https://www.loc.gov/bibframe/>, consultada el 8 de enero de 2016.

The Library of Congress, Network Development and Marc Standards Office. 2016. "Marc 21 Format for Authority Data. 1999 Edition Update No. 1 (October 2000) through Update No. 22 (April 2016)”. < http://www.loc.gov/marc/authority/ecadhome.html>, actualizada el 26 de abril de 2016, consultada el 2 de mayo de 2016.

The Library of Congress. 2015. "Standards at The Library of Congress". <http:// www.loc.gov/standards/>, actualizada el 13 de julio de 2015, consultada el 13 de octubre de 2015.

Tillet, Barbara B. 2004. "Authority control: State of the art and new perspectives". Cataloging and Classification Quarterly 38: 23-41.

Tillet, Barbara B. 1989. "Considerations for authority control in the online environment”. Cataloging and Classification Quarterly 9: 1-11.

Tosaka, Yuji y Jung-Ran Park. 2013. "RDA: Resource Description and Access. A survey of the current state of the art". Journal of The American Society for Information Science and Technology 64: 651-656.

Velluci, Sherry L. 2000. "Metadata and authority control". Library Resources and Technical Services 44: 33-43.

Veve, Marielle. 2009. "Supporting Name Authority Control in XML Metadata: A practical Approach at the University of Tenesse”. Library Research and Technical Services 53: 41-52.

W3C. 2015. "Semantic Web". <http://www.w3.org/RDF/>, actualizada en enero de 2015, consultada el 29 de junio de 2015.

Wolverton, R.E. 2006. "Becoming an authority on authority control: an annotated bibliography of resources”. Library Resources and Technical Services 50: 31-41.

Younger, Jennifer A. 1994. "After cutter: authority control in the twenty-first century”. Library Research and Technical Search 39: 133-141. 


\section{Normativa Utilizada}

Brickley, Dan y Libby Miller. 2014. "FOAF Vocabulary Specification 0.99. Namespace Document 14 January 2014. Paddington Edition”. <http://xmlns.com/foaf/ spec/>, actualizada el 14 de enero de 2014, consultada el $1^{\circ}$ de octubre de 2014.

Consejo Internacional de Archivos (CIA). 2004. ISAAR: Norma internacional sobre los registros de autoridad de archivos relativo a instituciones, personas y familias. Versión española de M. Elena Cortés Ruiz y Blanca Desantes Fernández. Madrid: Ministerio de Cultura, Secretaría General Técnica. <http://www.archivonacional.go.cr/pdf/ISAAR2-ES.pdf>, consultada el 5 de octubre de 2014.

Davis, Ian y Eric Vitiello. 2010. "Relationship: A vocabulary for describing relationships between people”. <http://vocab.org/relationship/>, actualizada el 19 de abril de 2010, consultada el 25 de octubre de 2014.

Dublin Core Metadata Initiative. 2012. "Dublin Core Metadata Terms". <http://dublincore.org/documents/2012/06/14/dcmi-terms/>, actualizada el 14 de junio de 2012, consultada el 14 enero de 2015.

García Melero, Justo (traductor). 2004. Directrices para registros de autoridad y referencias: anteriormente denominadas "Directrices para los asientos de autoridad y referencia" recomendadas por el Grupo de Trabajo para un Sistema Internacional de Autoridades, aprobadas por los Comités Permanentes de la Sección de Catalogación de la IFLA y de la Sección de Tecnología de la Información de la IFLA. Madrid: Ministerio de Cultura, Secretaría General Técnica. <http://www.ifla.org/files/cataloguing/garr/garr-es.pdf>, consultada el 29 de junio de 2014.

Patton, Glenn E. (ed.). 2009. Functional requeriments for authority data: A conceptual model. Múnich: K.G. Saur. <http://www.ifla.org/files/assets/cataloguing/frad/ frad_2009-es.pdf $>$, consultada el 23 de marzo de 2014.

Society of American Archivists. 2015. "EAC Encoded Archival Context, Corporate, Persons and Families”. <http://eac.staatsbibliothek-berlin.de/>, actualizada en enero de 2015, consultada el 11 de enero de 2015.

The Library of Congress. 2016. "Bibframe: Bibliographic Framework Iniciative". $<$ https://www.loc.gov/bibframe/>, actualizada el 6 de junio de 2016, consultada el 8 de enero de 2016.

The Library of Congress, Network Development and Marc Standards Office. 2016. "Marc 21 Format for Authority Data. 1999 Edition Update no. 1 (October 2000) through Update No. 22 (April 2016)”. Washington, D.C.: Library of Congress. <http://www.loc.gov/marc/authority/ecadhome.html>, actualizada el 26 de abril de 2016, consultada el 2 de mayo de 2016.

The Library of Congress, Network Development and Marc Standards Office. 2011. “mads: Metadata Authority Description. MADS 2.0". Washington, D.C.: Library of Congress. <http://www.loc.gov/standards/mads/>, actualizada el 6 de junio de 2011, consultada el 9 de junio de 2015.

The Library of Congress, Oficina de Desarrollo de Redes y Normas Marc. 2006. "Marc 21 Conciso para Datos de Autoridad. Ed. concisa de 2006. Actualización no. 1 (octubre de 2000)-Actualización no. 7 (octubre de 2006)". Washington, D.C.: The Library of Congress. <https://www.loc.gov/marc/authority/ecadspa. html>, actualizada el 4 de septiembre de 2008, consultada el 2 de mayo de 2014. 
The Library of Congress, Network Development and Marc Standards Office. 2004. "Marc xml, Marc 21 xml Schema". Washington, D.C.: The Library of Congress. $<$ http://www.loc.gov/standards/marcxml/>, actualizada el 30 de diciembre de 2004, consultada el $1^{\circ}$ de mayo de 2014.

W3C. 2014. "Resource Description Framework (RDF)". <http://www.w3.org/ RDF/>, actualizada el 15 de marzo de 2014, consultada el 29 de junio de 2014.

Para citar este texto:

Garzón-Farinós, Fernanda y Fernanda Peset. 2018. "Normativas interoperables sobre control de autoridades para bibliotecas digitales semánticas". Investigación Bibliotecológica: archivonomía, bibliotecología e información 32 (76): 167-188.

http://dx.doi.org/10.22201/iibi.24488321xe.2018.76.58018 